\title{
Do Transferred Patients Increase the Risk of Venous Thromboembolism in Trauma Centers?
}

Brian K. Yorkgitis, DO*

Brigham and Women's Hospital

Trauma, Burns, and Surgical Critical Care

75 Francis St.

Boston, MA 02115

BKY2009@gmail.com

P: 617-732-7715, F: 617-566-9549

Olubode A. Olufajo, MD, MPH

Brigham and Women's Hospital

Center for Surgery and Public Health

One Brigham Circle

1620 Tremont St. 4-020

Boston, MA 02120

David Metcalfe, LLB, MBChB

Brigham and Women's Hospital

Center for Surgery and Public Health

One Brigham Circle

1620 Tremont St. 4-020

Boston, MA 02120

Gally Reznor, MS

Brigham and Women's Hospital

Center for Surgery and Public Health

One Brigham Circle

1620 Tremont St. 4-020

Boston, MA 02120

Joaquim M. Havens, MD

Brigham and Women's Hospital

Trauma, Burns, and Surgical Critical Care

75 Francis St.

Boston, MA 02115

Zara Cooper, MD, MSc

Brigham and Women's Hospital

Trauma, Burns, and Surgical Critical Care

75 Francis St.

Boston, MA 02115

Ali, Salim, MD

Brigham and Women's Hospital

Trauma, Burns, and Surgical Critical Care

75 Francis St.

Boston, MA 02115

*Corresponding Author 


\begin{abstract}
Introduction: Trauma patients are often require initial stabilization followed by transfer for ongoing trauma care. Thus, administration of VTE prophylaxis is often delayed until admission to the receiving hospital. It is unclear if transfer status is a risk factor for VTE.
\end{abstract}

Methods: The National Trauma Database (NTDB) v6.2 was used to identify patients admitted to Level I and II trauma centers. Exclusions included patients on anticoagulation, $<18$ years, known VTE prior to trauma, or pregnant. Patients transferred were compared to non-transferred patients.

Results: Analysis included 736,374 patients with 189,166 (25.69\%) transferred patients within 24 hours of injury. Utilizing weighted measures, VTE was identified in 11,619 (1.50\%) patients. The VTE rate was significantly higher in the transferred group compared to the non-transferred group $(1.73 \%$ vs. $1.42 \%, \mathrm{p}=0.002)$ including DVT $(1.39 \%$ vs. $1.14 \%, \mathrm{p}=0.004)$ and PE ( $0.45 \%$ vs. $0.39 \%, \mathrm{p}=0.003)$. Multivariable analyses adjusting for patient-level risk factors demonstrated that transfer was associated with a higher likelihood of VTE (aOR 1.18; 95\% CI: 1.09-1.28, p=<0.001), PE (aOR 1.21; 95\% CI: 1.111.33, $\mathrm{p}=<0.001$ ), and DVT (aOR 1.17; 95\% CI: 1.07-1.28, $\mathrm{p}=0.0004)$.

Conclusion: Transfer status of trauma patients is a risk factor for VTE. Accepting a transferred patient results in an increased VTE risk and may not be reflective of the quality of care at the receiving facility. 


\section{BACKGROUND}

Venous thromboembolism (VTE) is the third most common cause of death among hospitalized trauma patients. ${ }^{1}$ Among trauma patients admitted with injuries, current VTE estimates vary between less than $1 \%$ and greater than $50 \%{ }^{2,3}$ However, the true incidence of VTE is difficult to determine as estimates vary depending on the extent to which patients are screened for VTE in the hospital. ${ }^{4}$ It is known that trauma itself can induce a hypercoagulable state ${ }^{5}$ and that this might be exacerbated by other injuryrelated considerations, such as the clinical need to minimize bleeding by omitting VTE chemoprophylaxis.

There has been much work to determine risk factors for VTE in trauma patients. ${ }^{6,7}$ To date, the impact of transfer status on VTE rates among trauma patients has not been described. There is some evidence that transfer between hospitals increases the odds of VTE among general surgery patients. ${ }^{8}$ The reason(s) for this finding are unknown, however it is plausible that inter-hospital transfers delay definitive management, prolong immobility, and may disrupt VTE prophylactic measures. The aim of this study was to explore this association. We hypothesized that patients transferred to trauma centers are at increased risk of developing VTE compared to those admitted directly to these centers.

\section{METHODS}

\section{Database and patient selection}

The National Trauma Database (NTDB) version 6.2 was queried for all adult (age $\geq 18$ years) admitted to level I trauma centers (LITC) and level II trauma centers (LIITC) between 2007 and 2012. The NTDB is the largest trauma database in the world, constituting patient records contributed from more than 900 participating centers across the United States. ${ }^{9} \mathrm{We}$ only examined patients included in the National Sample Program in order to have nationally representative estimates for our study. ${ }^{10}$ 
Patients were excluded if they were transferred after 24 hours from the presentation to the index receiving hospital, on anticoagulants at time of trauma, had a known VTE at time of trauma, or were pregnant.

\section{Variables}

The patient characteristics examined included transfer status, age, sex, length of stay (LOS), injury severity score (ISS), abbreviated injury scale (AIS) score, major head injury (defined as head AIS $\geq 3$, major limb injury (defined as extremity AIS $\geq 3$ ), major venous injury (defined by ICD-9 diagnosis codes of 900.1, 900.81, 901.2, 901.3, 902.1, 902.10, 902.11, 902.19, 902.3, 902.31, 902.32, 902.33, $902.34,902.42,902.52,902.54,902.56,902.82,903.02,904.2,904.3,904.42,904.52$, and 904.54$)^{11}$, and surgical procedures performed. Outcomes of interest included VTE status, hospital length of stay (LOS), ICU LOS, ventilator days, and in-hospital mortality. VTE was defined by ICD-9 diagnosis codes for deep venous thrombosis (DVT) (451.x, 453.x) and pulmonary embolism (PE) (415.1x). A secondary outcome was the in-hospital mortality rate after VTE event. Patient level variables for VTE risk were examined using the risk factors suggested by Knudson et al. ${ }^{6}$ These included age $\geq 40$ years, presence of severe extremity injury, presence of severe head injury, ventilator days $>3$, venous injury, major surgery (including operations on the nervous system [ICD-9 diagnosis codes 01.xx-05.xx], respiratory system [30.xx-34.xx], cardiovascular system [35.xx-39.xx], hematopeotic and lymphatic system, that is, spleen

procedures [40.xx-41.xx], digestive system [42.xx-54.xx], urinary system [55.xx-59.xx], and significant musculoskeletal procedures [77.xx, 78.xx, 81.xx, 83.xx, and 84.xx]). ${ }^{6,11}$

\section{Statistical analyses}


The primary outcome was VTE rate among trauma patients. A comparison on the rate of VTE during admission among transferred and non-transferred trauma patients was performed. A descriptive analysis of the patient characteristics was performed using Chi-square tests for categorical variables and Wilcoxon rank sum tests for continuous variables. Overall VTE, DVT, PE and mortality rates were calculated for the patients. Odds ratios were also calculated for each of these outcomes. An unadjusted logistic regression analysis was performed comparing VTE rates among transferred and non-transferred patients. VTE rates were similarly compared using the known risk factors earlier described. Multiple logistic regression analyses were performed to quantify the independent risk factors for VTE adjusting for factors associated with VTE in the unadjusted analysis and those previously identified by Knudson et al. ${ }^{6}$ Statistical analyses were undertaken using SAS 9.3 SURVEYLOGISTIC and SURVEYFREQ (SAS Institute Inc. Cary, NC) procedures to include NTDB weights, strata and clustering to create nationally representative estimates. Alpha was set on $P=0.05$. This study was approved by the Partners Healthcare's institutional review board (protocol \#2014P002162).

\section{RESULTS}

A total of 736,374 trauma patients were included in the study. There were 547,208 (74.3\%) patients that were directly admitted to a LITC or LIITC from the field and 189,166 (25.7\%) patients that were initially evaluated at an index receiving hospital and then transferred. Table 1 compares the patient characteristics between these two groups. Transferred patients were significantly older in years (mean, 51.7 vs. 47.4, p= $<0.001$ ), more injured overall (ISS 10.8 vs. $9.4, \mathrm{p}=<0.001)$, and had more severe head injury (28.2\% vs. $17.0 \%, \mathrm{p}=<0.001)$ when compared to the non-transferred group.

A total of 11,619 (1.50\%) VTE's were observed; 8,188 (1.42\%) in the non-transferred group and 3431 $(1.73 \%)$ in the transferred group $(\mathrm{p}=0.002)$ utilizing weighted measures (Table 2$)$. The proportions of patients with DVT and PE were each significantly higher among transferred patients than non-transferred patients ( $1.39 \%$ versus $1.14 \% \mathrm{P}=0.004$ and $0.45 \%$ versus $0.39 \% \mathrm{P}=0.003$, respectively). The overall mortality was $3.32 \%$, with $3.62 \%$ in the transferred group and $3.22 \%$ in the non-transferred group 
$(\mathrm{p}=0.015)$. The mortality among patients with VTE was also higher in transferred patients $(8.70 \%)$ when compared to non-transferred patients $(7.49 \%)(\mathrm{P}<0.001)$.

Independent Risk Factors for Venous Thromboembolism

On multivariable logistic regression analysis, hospital transfer was associated with increased odds of VTE $(\mathrm{aOR}=1.18 ; 95 \%$ CI 1.09-1.28, $\mathrm{p}=<0.001)$. Both DVT $(\mathrm{aOR}=1.17 ; 95 \%$ CI 1.074-1.28, $\mathrm{p}<0.001)$ and PE $(\mathrm{aOR}=1.21 ; 95 \%$ CI 1.11-1.33, $\mathrm{p}<0.001)$ were increased among transferred patients. Other major risk factors associated with VTE on multivariable analysis are demonstrated in Table 3. Among those patient that had a VTE and expired during their admission, there was no difference in mortality by transfer status after analysis $(\mathrm{OR}=1.062 ; 95 \%$ CI $0.951-1.186, \mathrm{p}=0.289)$.

\section{DISCUSSION}

The current study demonstrates that transferred trauma patients face an increased risk for developing a VTE. We have shown the risk of VTE increases $18 \%$ among patients requiring transfer. To our knowledge, this is the first study to demonstrate this association in trauma patients. This is an important factor that deserves further attention, as over $25 \%$ of trauma patients in our study transferred to receive their ongoing of trauma care. This work implicates an additional significant risk factor to the many previously described. ${ }^{6,7}$

Venous thromboembolism is a leading cause of in-hospital mortality and morbidity with its associated high costs. ${ }^{12}$ It is estimated that VTE is the cause of harm occurring every eight out of 1000 hospital stays and accounting for one in 17 in-hospital preventable deaths. ${ }^{13}$ Thus, it has become an important marker for quality. This has led to the development of the Patient Safety Indicator 12 (Postoperative Deep Vein Thrombosis and Pulmonary Embolism) by the Agency for Healthcare Research and Quality. The Patient Safety Indicators (PSIs) were intended to provide a set of measures for adverse events that occur in patients as a result of healthcare system exposure. PSIs are thought to be amenable to prevention by alterations at the system or provider level. ${ }^{14}$ VTE is one of the hospital-acquired conditions that has been 
targeted for an overall $40 \%$ reduction through a quality initiative led by the Center for Medicare and Medicaid Services. ${ }^{15}$ Strides have been made in the prevention of VTE in trauma patients through recognition of high risk patients and early administration of prophylactic measures. ${ }^{16,17}$ Unfortunately, even with aggressive prophylactic measures, patients continue to have VTE., ${ }^{4,18}$

With VTE used as a quality indicator, it can be linked to pay-for-performance initiatives. ${ }^{18,19}$ There are already concerns that the cost of providing comprehensive trauma services might outweigh the remuneration offered to hospitals designated as higher-level trauma centers. ${ }^{20}$ This is an important as policy moves to implement punitive measures for bench-marked VTE rates. A center that receives a high volume of transferred trauma patients may face penalties compared to a center with very low transferred patient volumes.

To explain the increase VTE rate in transferred patients, one factor may be due to a delay of prophylactic measures against VTE. From time of injury until evaluation and decision on chemical prophylaxis by the admitting trauma center, variable time periods elapse, during which VTE can develop. Additionally, patients are often immobile during the period of evaluation and transfer from the initial hospital to the admitting trauma center may increase their risk for VTE. Through development of relationships between referral trauma centers and hospitals that commonly transfer trauma patients to these centers, patients could be identified that may benefit from administration of prophylactic measures prior to transfer.

There are a number of limitations of this study that need to be pointed out. First, we recognize retrospective use of this database precludes the knowledge of specific patient and hospital level factors. Under reporting of complications is a well-recognized flaw. ${ }^{4}$ Utilizing comparison of patients meeting the inclusion criteria at centers reporting to NTDB hopefully mitigates this error. Some important factors for increased risk of VTE that could not be ascertained include which modality of prophylaxis, if any that were used for the patients. Each trauma center may have variations in their VTE prophylaxis protocols. Included in this variation may be timing of initiation, agent(s) used, and adherence rate to the facilities' 
protocol in the administration of these modalities. Through the use of NTDB, we cannot know the rate of missed administration of these measures, placing the patients at higher risk for VTE. ${ }^{21}$ It has been examined by several studies, that some centers may utilize screening modalities to detect asymptomatic VTE. ${ }^{4,18}$ Since individual center's practice on screening is unavailable, a screening bias may be present which we did not account for. This screening bias may be minimized by comparing patients that were directly admitted to a trauma center, implying equal practice for all patients regardless of transfer status to a particular center.

\section{CONCLUSION}

Trauma patients transferred for their ongoing trauma care have an increased risk of VTE. Further prospective studies may assist in identifying transferred patients at the highest risk and specific interventions to decrease VTE rates. Centers that accept high volumes of transferred trauma patients may face unfairly low quality ratings due to factors beyond their control. It is therefore necessary to take note of this potential consequence in comparing hospital performance. 


\section{REFERENCES}

1. Shackford SR, Mackersie RC, Holbrook TL, Davis JW, Hollingsworth-Frilund P, Hoyt DB, et al. The epidemiology of traumatic death: a population-based analysis. Arch Surg. 1993;128:571-575.

2. Huseynova K, Xiong W, Ray J et al. Venous Thromboembolism as a Marker of Quality of Care in Trauma. J Am Coll Surg. 2009;208:547-552.

3. Geerts WH, Code KI, Jay RM, Chen E, Szalai JP. A prospective study of venous thromboembolism after major trauma. N Engl J Med. 1994;331:1601-1606.

4. Haut ER, Noll K, Effron DT, et al. Can Increased Incidence of Deep Vein Thrombosis (DVT) be Used as a Marker of Quality Care in the Absence of Standardized Screening? The Potential Effect of Surveillance Bias on Reported DVT rates after Trauma. J Trauma. 2007;63(5):1132-1137.

5. Selby R, Geerts W, Ofosu FA, Craven S, et al. Hypercoagulability after trauma: hemostatic changes and relationship to venous thromboembolism. Thromb Res. 2009;124-3:281-287.

6. Knudson MM, Ikossi DG, Khaw L, et al. Thromboembolism after trauma: an analysis of 1602 episodes from the American College of Surgeons National Trauma Data Bank. Ann Surg. 2004;240-3:490-6; discussion 6-8.

7. Rogers F, Cippolle M, Velmahos G, et al. Practice Management Guidelines for the Prevention of Venous Thromboembolism in Trauma Patients: The EAST PRactice Management Guidelines Work Group. J Trauma. 2002;53(1):142-164.

8. Lucas DJ, Ejaz A, Haut ER, et al. Interhospital transfer and adverse outcomes after general surgery: implications for pay for performance. J Am Coll Surg. 2014;218-3:393-400.

9. American College of Surgeons. National Trauma Data Bank: NTDB Research Data Set Admission Year 2010, Annual Report. Chicago, IL:American College of Surgeons; 2010. 
10. Goble S, Neal M, Clark DE. Et al. Creating a nationally representative sample of patients from trauma centers. J Trauma Acute Care Surg. 2009;67(3):637-644.

11. Haut ER, Chang DC, Pierce CA, et al. Predictors of Posttraumatic Deep Vein Thrombosis (DVT): Hospital Practice Versus Patient Factors- An Analysis of the National Trauma Data Bank (NTDB). J Trauma. 2009;66:994-1001.

12. Fernandez MM, Hogue S, Kwong WJ. Review of the cost of venous thromboembolism. Clinicoecon Outcomes Res. 2015;7:451-462.

13. Center for Disease Control and Prevention. CDC Grand Rounds: Preventing Hospital-Associated Venous Thromboembolism. Morbidity and Mortality Weekly Report. 2014;63(9):190-193.

14. Centers for Medicare and Medicaide Services. Patnership forPatients. Available at: https://partnershipforpatients.cms.gov/. Accessed February 2, 2015.

15. Agency for Healthcare Research and Quality. Perioperative Pulmonary Embolism or Deep Vein Thrombosis Rate.

http://qualityindicators.ahrq.gov/Downloads/Modules/PSI/V50/TechSpecs/PSI_12_Perioperative_Pulmon ary_Embolism_or_Deep_Vein_Thrombosis_Rate.pdf. Accessed November 3, 2015.

16. Paffrath T, Wafaisade A, Lefering R, et al. Venous thromboembolism after severe trauma: Incidence, risk factors and outcome. Injury. 2010;41:97-101.

17. De Virgilio C, Kim JJ. More Evidence That the Use of Venous Thromboembolism Rates as Hospital Quality Measures May Be Off the Mark. J Am Med Assoc Surg. 2015;150(8):721.

18. Ju MH, Chung JW, Kinnier CV, et al. Association between hospital imaging use and venous thromboembolism events rates based on clinical data. Ann Surg. 2014;260-3:558-64; discussion 64-6. 19. Michtalik HJ, Carolan HT, Haut ER,et al. Use of provider-level dashboards and pay-for-performance in venous thromboembolism prophylaxis. J Hosp Med. 2015;10-3:172-8. 
20. Friedman, E. The Roller-Coaster Supple of Burn and Trauma Care. Hospitals \& Health Networks. Available at: http://www.hhnmag.com/articles/3589-the-roller-coaster-supply-of-burn-and-traumacare\#.VuUPthGu9II.email. Accessed March 1, 2016.

21. Louis GZG, Geraci T, Anderson R, et al. Correlation of missed doses of enoxaparin with increased incidence of deep vein thrombosis in trauma and general surgery patients. JAMA Surg. 2014;149(4):365370. 
Table 1. Comparison of Characteristics of Trauma Patients admitted to Level I and II Trauma Centers

\begin{tabular}{|c|c|c|c|}
\hline \multirow[t]{2}{*}{ Characteristic } & \multicolumn{2}{|c|}{ No. of Patients (Weighted Proportion) } & \multirow[b]{2}{*}{$\mathbf{p}$} \\
\hline & Non-transferred & Transferred & \\
\hline Number of patients & 547,208 & 189,166 & - \\
\hline Male & $360,225(64.92)$ & $125,346(64.53)$ & 0.310 \\
\hline$\geq 40$ years old & $313,557(59.0)$ & $121,439(66.91)$ & $<0.001$ \\
\hline Major head injury & $103,072(17.03)$ & $53,087(28.15)$ & $<0.001$ \\
\hline Major limb injury & $23,160(3.78)$ & $7,628(3.58)$ & 0.235 \\
\hline Surgery & $179,427(34.39)$ & $65,868(33.71)$ & 0.656 \\
\hline Major venous injury & $3,896(0.62)$ & $677(0.28)$ & $<0.001$ \\
\hline ISS $*$ & 9.39 & 10.81 & $<0.001$ \\
\hline Hospital LOS*, days & 5.84 & 6.32 & 0.001 \\
\hline ICU LOS*, days & 5.78 & 5.44 & 0.052 \\
\hline Ventilator days*, days & 6.65 & 6.65 & $>0.999$ \\
\hline
\end{tabular}

ISS- Injury Severity Score, LOS-length of stay, VTE- venous thromboembolism, DVT-deep venous thrombosis, PE- pulmonary embolism *Mean estimates 
Table 2. Univariate Analysis of Outcomes among Trauma Patients based on Transfer Status

\begin{tabular}{|lccc|}
\hline \multirow{2}{*}{ Outcome } & \multicolumn{2}{c}{$\begin{array}{c}\text { No. of Patients } \\
\text { (Weighted Proportion) }\end{array}$} & $\begin{array}{c}\text { Odds Ratio } \\
\text { (95\% Confidence Interval) }\end{array}$ \\
\cline { 2 - 3 } & Non-transferred & Transferred & \\
\hline VTE & $8188(1.42)$ & $3431(1.73)$ & $1.22(1.17-1.27)$ \\
DVT & $6445(1.14)$ & $2704(1.39)$ & $1.22(1.16-1.27)$ \\
PE & $2234(0.39)$ & $933(0.45)$ & $1.21(1.12-1.31)$ \\
Mortality & $19152(3.22)$ & $7105(3.62)$ & $1.13(1.11-1.14)$ \\
\hline
\end{tabular}

VTE- venous thromboembolism, DVT-deep venous thrombosis, PE- pulmonary embolism 
Table 3. Multivariate Logistic Regression Analysis of Factors associated with Venous Thromboembolism among Trauma Patients

\begin{tabular}{|lcc|}
\hline Risk Factor & aOR & 95\% CI \\
\hline Transfer & 1.18 & $1.09-1.28$ \\
Male sex & 1.27 & $1.22-1.32$ \\
Age $\geq 40$ years & 1.90 & $1.81-2.00$ \\
Surgical procedure & 3.76 & $3.37-4.19$ \\
Major limb injury & 1.19 & $1.09-1.29$ \\
Major head injury & 1.23 & $1.12-1.34$ \\
Venous injury & 1.88 & $1.50-2.36$ \\
LOS (days) & 1.04 & $1.04-1.04$ \\
ISS & 1.03 & $1.03-1.03$ \\
\hline
\end{tabular}

ISS- injury severity score, LOS-length of stay, aOR- adjusted Odds Ratio (adjusted for all variables in the table) 